\title{
Mitochondria as a biomarker for IVF outcome
}

\author{
Julia Kim ${ }^{1,2}$ and Emre Seli ${ }^{1,3}$ \\ ${ }^{1}$ IVI/RMA of New Jersey, Basking Ridge, New Jersey, USA, ${ }^{2}$ Sidney Kimmel Medical College of Jefferson University, \\ Philadelphia, Pennsylvania, USA and ${ }^{3}$ Yale School of Medicine, New Haven, Connecticut, USA
}

Correspondence should be addressed to E Seli; Email: emre.seli@yale.edu

\begin{abstract}
Mitochondria play an essential role in generating energy for embryo development and maintaining embryo metabolism through key cellular functions including ion homeostasis, amino acid metabolism, glycolysis, fatty acid metabolism, signal transduction and apoptotic regulation. Recent literature suggests that mitochondrial content and function may be related to implantation success and embryo viability. Some studies have linked increased levels of mitochondrial DNA to aneuploidy, advanced maternal age and euploid blastocyst with implantation failure, while others have failed to demonstrate similar findings. This review aims to provide an overview of the current literature surrounding the possibilities of using mitochondria as an additional biomarker for infertility treatment outcome and summarize the reasons as to why there are inconsistencies in these studies.

Reproduction (2019) 157 R235-R242
\end{abstract}

\section{Introduction}

The field of reproductive medicine has made great strides over the past several decades in the ability to select an embryo generated by in vitro fertilization (IVF) for its successful reproductive potential. Initially, embryologic morphology and cleavage rates formed the sole bases for selection of embryos suitable for transfer; however, while these methods led to an improvement in outcomes, the majority of transferred embryos still failed to implant and yield ongoing pregnancies (Gardner \& Schoolcraft 1999, Toner 2002). With the dawn of preimplantation genetic testing for aneuploidy (PGT-A), the ability to transfer only euploid embryos contributed to a noticeable increase in live birth rates (Forman et al. 2014). Despite these advances, selection and subsequent transfer of euploid embryos do not always result in ongoing pregnancies, highlighting that there is more to embryonic viability than just chromosomal normalcy (Harton et al. 2013). As studies in animal models suggest a strong association between mitochondria and reproductive function, there has been an increasing interest in human reproductive literature toward mitochondria and the use of mitochondrial parameters to supplement currently existing selection methods with the goal of prognosticating viability of preimplantation embryos in IVF (Ramalho-Santos et al. 2009, Wells 2017, Wang et al. 2018).

\section{Mitochondrial DNA}

The mitochondrion is a semi-autonomous organelle that plays an integral role in key cellular functions including
ATP production, regulation of apoptosis, calcium homeostasis and generation of reactive oxygen species (ROS). Mitochondria are double-membrane bound organelles thought to be derived from endosymbiotic $\alpha$-purple bacteria profiting from host oxygen (Taanman 1999). The circular mtDNA genome is composed of approximately $16.6 \mathrm{~kb}$ of double-stranded DNA (Fig. 1). The dual strands of mtDNA encode a total of 37 genes that translate to 22 tRNAs, 2 rRNAs and 13 protein polypeptides. These 13 proteins function as part of the electron transport chain (ETC) that facilitates ATP production through oxidative phosphorylation (OXPHOS) with the remainder of the requisite proteins encoded by nuclear DNA (Anderson et al. 1981).

Until very recently, it was thought that mitochondria almost entirely stemmed from maternal inheritance, with paternal mitochondria being essentially undetectable after the four-cell stage in a fertilized embryo (Cummins 2000). However, a recent study found evidence of paternal mtDNA transmission coexistent with maternal inheritance in three unrelated families, challenging the long-held assumption of sole maternal mtDNA transmission in humans. While this discovery marks a sentinel change in the dogma of mitochondrial inheritance, the authors emphasize that biparental inheritance is still quite rare (Luo et al. 2018). Previous research hypothesized that the essentially exclusive maternal inheritance is likely an evolutionary advantage to avoid the bequeathal of sperm mitochondrial DNA that has suffered the deleterious effects of free radicals (Aitken et al. 1998). Spermatozoa create their own ROS as part of normal sperm capacitation; however, excess ROS can cause lipid peroxidation that further 

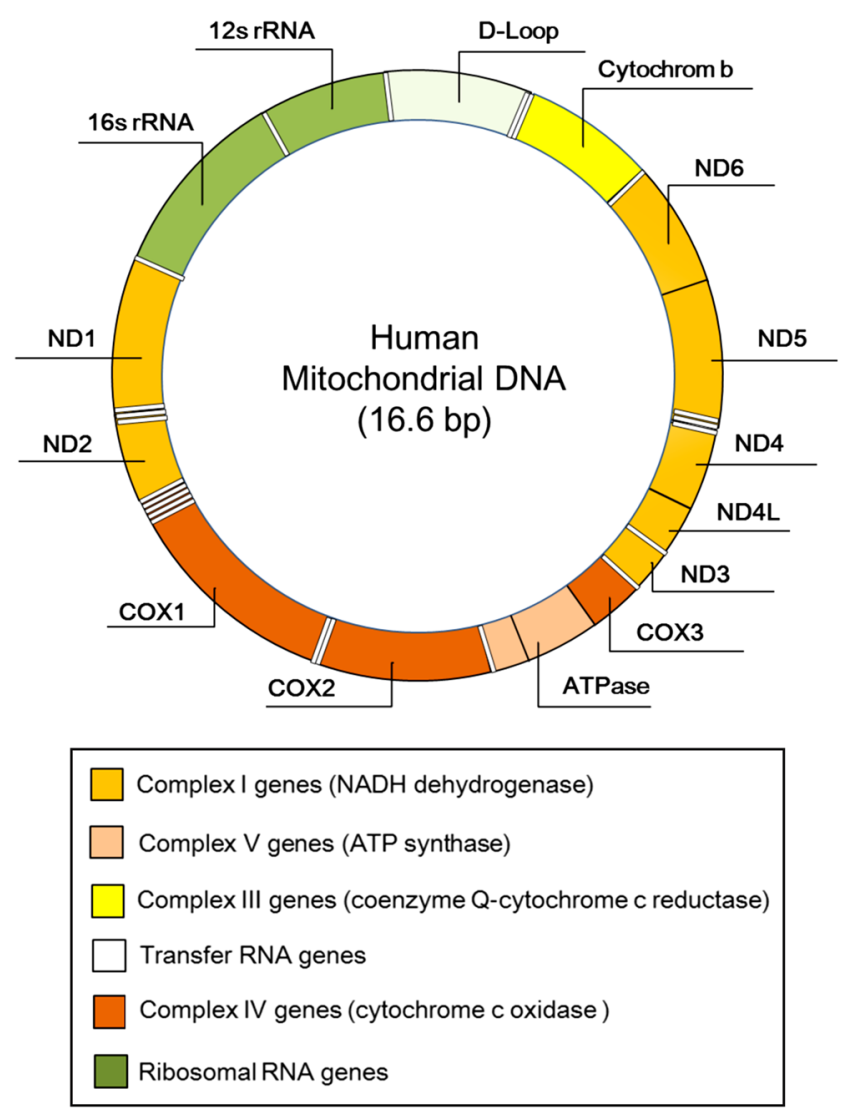

Figure 1 Mitochondrial DNA. The circular mitochondrial DNA is approximately $16.6 \mathrm{~kb}$ and double stranded. It contains a total of 37 genes encoding 22 tRNAs, 2 rRNAs and 13 protein polypeptides, all of which are part of the electron transport chain.

stimulates ROS generation from the mitochondrial electron transport chain. This triggers elevated levels of oxidative stress that leads to mtDNA damage (Aitken 2017). Another hypothesis to explain sperm mtDNA's degradation after fertilization is sequestration of sperm mtDNA to specific embryonic regions separate from the inner cell mass (Birky 1995). A ubiquitin-mediated process leading to targeted sperm mtDNA degradation upon fertilization has also been proposed in a bovine model (Sutovsky et al. 1996).

Oogonia in early fetal development initially contain approximately 200 mitochondria, a number that continuously increases as the oocyte proceeds through meiosis, reaching a zenith of 100,000 mitochondria and 50,000-550,000 mtDNA copies at metaphase II. The mature oocyte has some of the highest mitochondrial content amongst human cells, likely due the great energy requirements of fertilization and early embryonic development (Reynier et al. 2001, May-Panloup et al. 2005). Additionally, bovine oocytes with higher mtDNA copy numbers appear more likely to mature (LamasToranzo et al. 2018). Previous research in ovine oocytes demonstrated that mtDNA copy number increases from the germinal vesicle (GV) to metaphase II (MII) stages of development (Cotterill et al. 2013). However, a recent study looking at mouse and human oocytes found that mtDNA copy number decreased from the GV to MII stages in both species in younger and older maternal age groups; the authors suggested that the contrasting trend in mtDNA copy number may be attributed to species-specific mechanisms of oocyte mitochondrial degradation during the maturation process (Pasquariello et al. 2018).

\section{Aging and mtDNA}

It is well known that female reproductive potential steadily decreases with age: as early as 1986, Menken et al. reported the rate of involuntary childlessness in women aged 20-24 years as $6 \%$, in women aged $30-34$ up to $15 \%$ and women $40-44$ as high as $64 \%$ (Menken et al. 1986). This trend has been mirrored in patients' undergoing IVF treatment. The 2016 data from the Society of Assisted Reproductive Technology (SART) revealed that patients using their own oocytes had live birth rates per started IVF cycle of $47.6 \%$ in women under 35 years, $34.8 \%$ in women aged $35-37$, $21.8 \%$ in women aged $38-40$ years, $11.2 \%$ in women aged $41-42$ years and as low as 3.3\% in women over 42 years (SART 2016).

While mtDNA levels can vary amongst oocyte cohorts, de Boer et al. noted that average mtDNA levels decrease with advancing maternal age and diminished ovarian reserve (DOR) (de Boer et al. 1999). One study identified that the range of mtDNA copy number in patients with DOR undergoing IVF was approximately 100,000 copies $( \pm 99,000)$, whereas that of patients with normal ovarian reserve was approximately 318,000 copies $( \pm 184,000)$ (May-Panloup et al. 2005). It has been hypothesized that the decreased mtDNA copy numbers in the DOR patients may be the consequence of insufficient mitochondrial synthesis or aberrant cytoplasmic maturation in oocytes. Reynier et al. proposed a defect in mitochondrial biogenesis after observing that many of the DOR patients' oocytes had abnormal cytoplasmic maturity, noting that a large surge of mitochondrial content occurs during the final maturation of the oocyte (Reynier et al. 2001). Oocyte mitochondria also demonstrate structural and functional alterations associated with aging: mitochondrial swelling, increased vacuolization and alteration of cristae have been noted in older patients (Muller-Hocker et al. 1996). Additionally, mitochondrial activity as measured by mitochondrial membrane potential has also been noted to change in older humans (Wilding et al. 2001). Babayev et al. identified age-associated differences in expression of genes linked to mitochondrial function and oxidative stress, as well as a decreased mtDNA copy number in mouse oocytes (Babayev et al. 2016). Specifically, mouse genes responsible for ATP production were upregulated in younger murine oocytes compared 
to those in older oocytes; additionally, researchers found genes protective against stress-related cellular damage to be downregulated in older mouse oocytes (Hamatani et al. 2004).

It is important to recognize that mtDNA is inherently more prone to mutations compared to nuclear DNA, due to its proximity near the respiratory chain with greater exposure to ROS, as well as its deficiency of the protective histones and repair mechanisms that nuclear DNA retains (Lynch et al. 2006). The nearly 25 times higher mutation rate of mtDNA leads to progressively higher numbers of deletions and point mutations, even in healthy older patients (Cortopassi \& Arnheim 1990). The link between mtDNA and aging has been further emphasized in studies investigating mutations in mtDNA polymerase (POLGA). Mice lacking a nuclearencoded subunit of POLGA have higher numbers of mtDNA mutations as well as deletions; they also possess a prematurely older phenotype that confers infertility in both male and female mice. These phenotypic changes included weight loss, reduction in subcutaneous fat, osteoporosis, anemia and cardiomyopathy (Trifunovic et al. 2004). Human studies have similarly demonstrated that some individuals bearing POLGA mutations experience premature menopause (Luoma et al. 2004, Day et al. 2015).

Although prior research has identified decreased number of mtDNA levels in the oocytes of older and DOR patients, blastocysts from this same population demonstrated increased levels of mtDNA (May-Panloup et al. 2005, Diez-Juan et al. 2015). Fragouli et al. found that real-time PCR demonstrated a statistically significant increase in the amount of mtDNA in 154 blastocysts from an older reproductive population (average age 39.8 years) compared to that of 148 blastocysts from younger population (average age 34.8 years). This trend also held true when euploid and aneuploid embryos were analyzed independently (Fragouli et al. 2015). The prevailing thought regarding this relationship has been that suboptimal circumstances such as those created by aneuploid embryos or advanced maternal age create an environment that triggers a state of stress leading to increased mitochondrial DNA synthesis. This represents a deviation from the ideal 'quiet embryo' environment proposed to be the norm for optimal embryo development (Leese 2002). It follows that increased mtDNA levels may represent a 'stressed' embryo's attempt to create more ATP to surmount the atypical conditions of aneuploidy or advanced maternal age (Victor et al. 2017). Prior studies have found that aneuploid embryos are associated with slower development and arrest compared to euploid embryos; however, a causal relationship has not yet been identified between stress and chromosomal abnormality (Kroener et al. 2012, Kort et al. 2015). It is also unknown as to whether embryonic aneuploidy alters cellular metabolism or oxidative homeostasis in human embryos; this is unfortunately also a difficult question to study in animal models given their low rates of aneuploidy. It is possible that the various processes of embryo culture inherent to IVF could also affect mtDNA copy number, but to our knowledge, this has not been studied.

\section{Mitochondrial DNA copy number as a biomarker in IVF}

In examining the correlation between mitochondria and embryonic competence, mtDNA copy number emerged as an indirect way of measuring embryonic function, leading to subsequent interest in mtDNA copy number's use as a biomarker of embryonic competence (DiezJuan et al. 2015, Fragouli et al. 2015). Initial evidence indicated that increased mtDNA copy numbers are associated with aneuploid embryos as well as with euploid blasts that fail to implant; however, there have been other studies contradicting these findings (Treff et al. 2017, Victor et al. 2017, Klimczak et al. 2018).

The earliest study investigating the link between mtDNA copy number and embryonic viability by Fragouli et al. investigated cleavage-stage embryos and blastocysts through a combination of array-comparative genomic hybridization $(\mathrm{aCGH})$, real-time quantitative polymerase chain reaction (qPCR) and next-generation sequencing (NGS) approaches (Fragouli et al. 2015). The study's validation protocol included use of NGS to re-affirm mtDNA copy number in 23 blastocysts initially quantified by qPCR. The day 3 embryos had a single blastomere analyzed, whereas the day 5 embryos yielded 5-10 blastomeres from trophectoderm biopsies for analysis. In the first part of the study, a total of 39 cleavage-stage embryos and 302 blastocysts underwent aCGH, with another 38 blastocysts analyzed by NGS. All of the day 3 embryos were euploid and subsequently underwent embryo transfer. Of the blastocysts, 203 of the aCGH group and 14 of the NGS group were euploid; 131 of these euploid blastocysts were transferred. qPCR was utilized to quantify mtDNA copy number by amplifying the mtDNA sequence responsible for coding the $16 \mathrm{~S}$ ribosomal RNA, using the genomic DNA Alu sequence as a reference for normalization. Cleavage stage embryos from older women had lower mtDNA copy numbers, but had no significant association with implantation potential. However, the study's blastocyst analysis revealed a significantly higher mtDNA copy number in aneuploid blastocysts, blastocysts from older women and euploid blastocysts that failed to implant. The authors also determined that no pregnancies occurred when mtDNA copy number crossed a relative threshold. This translated to $47.2 \%$ of study population with successful implantation of transferred blastocysts, but $0 \%$ successful implantation of embryos with increased mtDNA copy numbers above the threshold. A subsequent prospective study 
by the same group again found that euploid blasts with elevated mtDNA copy numbers failed to result in ongoing pregnancies (Fragouli et al. 2017).

A large multi-center study subsequently examined mtDNA copy number in 1505 blastocysts from 490 couples who had previously undergone genetic testing for aneuploidy at 35 various clinics (Ravichandran et al. 2017). mtDNA copy number was analyzed by $\mathrm{qPCR}$ and investigators were blinded to prior implantation results, thus allowing predictions to be made regarding implantation success based upon the qPCR results. Using the previously established threshold from Fragouli's initial study, 139 out of the 1505 blastocysts $(9.2 \%)$ were found to have mtDNA levels exceeding this threshold and were hypothesized to have decreased implantation. At the time of publication, 282 euploid blastocysts had undergone endometrial transfer with 185 successfully implanting $(65.6 \%)$. Of the 282 blastocysts transferred, 249 had normal levels of mtDNA and 185 of these resulted in successful pregnancy, yielding a higher implantation rate of $74.3 \%$ amongst euploid embryos with normal mtDNA copy numbers. The 33 remaining embryos that had elevated levels of mtDNA all failed to result in successful pregnancies. It is intriguing to note that amongst the 35 different clinics, some centers had far more embryos with elevated mtDNA levels than others. While the mechanism behind the discrepancy remains unclear, it has been conjectured that certain external factors that are known to vary between labs may exert a certain degree of 'stress' manifested in higher levels of embryonic mtDNA (Wells 2017).

Another study by Diez-Juan et al. published shortly after Fragouli's initial study examined 205 cleavagestage embryos and 65 blastocysts, similarly testing a single blastomere from day 3 embryos and 5 to 10 blastomeres from blastocysts for euploidy and mtDNA copy number (Diez-Juan et al. 2015). This study utilized $\mathrm{aCGH}$ and $\mathrm{qPCR}$ for its analysis, but normalized qPCR data to actin, which bears a single copy sequence at risk of error due to allele dropout (ADO) particularly in the event of single-cell analysis (Seli 2016). Like Fragouli's initial study, Diez-Juan concluded that increased mtDNA copy number is associated with lower implantation rates in euploid embryos; moreover, this finding was noted in both blastocysts as well as cleavage stage embryos. Diverging from the initial study, however, the authors failed to identify a correlation between mtDNA copy number and maternal aging in either day 3 or day 5 embryos.

A subsequent study by Treff et al. examined sibling embryos in which 187 known male-female blastocyst DETs occurred, resulting in 69 singleton live-births (Treff et al. 2017). These blastocysts' ploidy results were analyzed by comprehensive chromosome sequencing (CCS) and mtDNA copy number quantification was performed by qPCR. The utilization of DET allowed researchers to investigate whether the embryo that resulted in a live birth had different amounts of mtDNA than the opposite sex nonviable sibling embryo. The study did not identify any differences in relative mtDNA copy number between the sibling embryo that resulted in a live birth and the other that did not. The authors noted that the embryos in the 90th percentile for mtDNA copy number had slightly decreased implantation rates, but still were as high as $48.5 \%$, representing a marked difference from the $0 \%$ implantation of embryos in the 90th percentile of Fragouli's initial study.

Victor et al. echoed these findings in a large study investigating 1396 embryos initially biopsied for PGT-A purposes (Victor et al. 2017). The authors did not identify any statistically significant differences in mtDNA copy number amongst embryos regarding ploidy, maternal age or implantation rates. As in some of the prior studies, PGT-A was performed by NGS and the mtDNA copy number was assessed by qPCR. Using NGS, results were in the form of multiple DNA sequences across the nuclear DNA genome. These genomic fragments were mapped and counted to calculate the amount of DNA in each chromosome to project a chromosome copy number. These NGS data also contained a smaller portion of mtDNA sequences, permitting the calculation of a mitochondrial-tonuclear DNA ratio to approximate a relative measure of mtDNA cellular content. This study also uniquely devised a correction factor to account for differences in nuclear DNA between female and male embryos as well as any discrepancies between euploid and aneuploid embryos. This adjustment was conducted with the goal of improving accuracy that may have been hindered by intrinsic genomic variation. The authors conducted additional analyses with PCR as opposed to NGS, using prior WGA products. Given the high accuracy of qPCR's ability to quantify nucleic acid number, this study represents another strong argument for the lack of association between mtDNA and blastocyst viability.

Most recently, a study by Klimczak et al. looking at 1510 blastocyst biopsies identified that mtDNA copy number correlated with the highest grade and lowest grade embryos in terms of morphology, but this association did not hold amongst mid-grade embryos (Klimczak et al. 2018). Furthermore, when the analysis examined solely euploid embryos, there was no longer an association between mtDNA copy number and embryo morphologic grade. The authors also noted no statistically significant differences in mtDNA copy number between embryos that implanted and those that did not. Additionally, investigators did not identify the differences in mtDNA copy number between embryos resulting in ongoing pregnancies and those that failed. They did find that amongst blastocysts, day 5 embryos had statistically significantly higher levels of 
mtDNA compared to day 6 embryos, but lower rates of aneuploidy and higher quality scores according to the Gardner blastocyst grading system. This study utilized NGS and qPCR as in prior studies for their analysis.

\section{Methodologic considerations}

With the literature practically split regarding the significance of mtDNA copy number in relation to embryo viability, it is important to identify the potential causes of the disparities. It has been proposed that the number of cells in a single trophectoderm biopsy widely varies among samples (Wells 2017). It follows that a greater number of cells would result in a greater number of mtDNA copies, which is why all prior studies used nuclear DNA to normalize results and allow calculation of relative mtDNA per cell. However, some studies have used only single nuclear DNA reference sequences, whereas others have normalized their data against multiple nuclear DNA sequences (Diez-Juan et al. 2015, Fragouli et al. 2015). Utilizing only a single reference sequence exposes the risk of allele dropout and amplification failure at rates as high as $50-60 \%$ in five-cell biopsy specimens (Wells 2017). Amplification failure of nuclear DNA would result in decreasing the amount available for comparison to mtDNA, potentially falsely elevating the normalized amounts of mtDNA. In studies using large number of samples, method of normalization between plates also poses a technical challenge, requiring standards or samples that are repeated in each plate with high consistency.

Other potential factors that may affect the accurate measurement of mtDNA include compromised integrity of DNA due to prolonged storage time. Both mitochondrial and nuclear DNA have been found to demonstrate evidence of degradation after a matter of months, raising question as to validity of mtDNA copy number and nuclear DNA content in some study specimens that had been stored for multiple years (Piyamongkol et al. 2003).

All in all, an explanation regarding the cause of differing conclusions from various centers has not been elucidated as of yet. It is important to note that conflicting studies regarding the utility mtDNA have been proof-of-concept studies, which compare samples to each other in a similar cohort (Diez-Juan et al. 2015, Fragouli et al. 2015, Victor et al. 2017). Treff et al.'s sibling study may be seen as a proxy to a non-selection study to investigate this question, yet found no clear clinical benefit to identification of mtDNA levels, as it did not affect the outcomes (Treff et al. 2017). While sibling and non-selection studies can assist with validating proof-ofconcept studies, only a randomized controlled trial can truly demonstrate the benefit to outcomes.

A commercially available assay known as MitoScore has become available to patients, claiming to select embryos with higher implantation potential to improve pregnancy rates and reproductive success (iGenomix Mitoscore 2018). Until further validation regarding the validity of mtDNA copy number has been met, the prognostic utility of this test cannot be entirely guaranteed.

\section{Alternatives to measuring embryonic mtDNA copy number}

Besides examining direct samples from blastocyst biopsies, a number of studies have investigated mtDNA content on other specimens collected during IVF such as cumulus cells and spent embryo culture media (Desquiret-Dumas et al. 2017, Hammond et al. 2017). Stigliani et al. demonstrated that embryos with inferior morphologic quality had increased levels of both genomic and mitochondrial DNA in embryo culture media (Stigliani et al. 2013). These findings were contradicted by the same authors in a subsequent study revealing an increase in the ratio of relative mtDNA to genomic DNA in day 3 embryo spent culture media correlated with increased rates of blastocyst formation and implantation (Stigliani et al. 2014). These findings may be explained by subsequent research that culture media may be contaminated by maternal cumulus cell DNA, limiting accurate evaluation of nuclear and mtDNA in these samples (Hammond et al. 2017). Another study identified that elevated levels of mtDNA in cumulus granulosa cells, which surround a developing oocyte and coordinate development, reflect day 3 embryos with high morphology grades (Desquiret-Dumas et al. 2017). These are consistent with the aforementioned literature looking at mitochondrial DNA content in older oocytes and corroborate that higher levels of mtDNA in the earlier stages of development is associated with improved viability, representing a reversal from the 'less is better' theory put forth in some of the blastocyst mtDNA literature.

Besides direct measurement of cellular DNA content, it is worth mentioning that there have also been forays into assessment of mitochondrial function through other methods. One such study utilized Fluorescence Lifetime Imaging Microscopy (FLIM) as a noninvasive means of detecting mitochondrial dysfunction in murine oocytes (Sanchez et al. 2018). This technique measures inherent NADH and FAD autofluorescence in cells. In the study, oocytes with metabolic dysfunction (obtained from mice with a germline deletion of the mitochondrial protease, Clpp) were imaged with FLIM and compared to oocytes from wild-type mice. The fluorescence intensities as well as NADH/FAD lifetimes between the knockout mice and wild-type controls were noted to be distinct and reflective of differences in metabolic function. They compared these observations to mtDNA copy number performed by qPCR on the same oocytes and found only slight 
variations in mtDNA copy number between knockout and wild-type samples. While further validation is required for this technique in its ability to accurately detect mitochondrial function in human models and how it relates to embryonic viability, it represents another potential method that may possess less room for variation compared to mtDNA copy number.

\section{Conclusions}

There still remains a lack of clarity regarding the relationship between mitochondrial function and embryonic viability. It seems counterintuitive that mitochondria, as the organelles responsible for the production of energy, in excess could lead to deleterious effects in implantation or development. It has been proposed that an abnormally high amount of mtDNA may represent a compensatory response to mitochondrial mutations leading to reductions in energy synthesis, so although the actual numbers of mitochondrial may be initially normal, their actual function is decreased (Monnot et al. 2013). As a consequence, the cell may be increasing the number of mitochondria to create enough ATP to sustain embryonic development. Another explanation goes along with the 'quiet embryo hypothesis', which states that normal embryos demonstrate less active metabolic activity. Embryos with decreased viability potential such as those that are aneuploid or from older oocytes with less mitochondria - and thus potentially decreased energy-forming capacity - may be under a certain amount of cellular stress that stimulates an increase in mitochondrial production to combat the toll of these conditions (Leese 2002).

Given that not all studies have been able to link an association between increased mtDNA content and decreased implantation or embryonic viability, it is clear that further research into this area is still merited. Aside from answering the question regarding the association itself, identification of the best methodologies to measure mtDNA content accurately and decreasing room for error is also necessary. Given the wide variability between embryo cohorts and amongst clinics in proportion of embryos, a multi-center prospective randomized controlled study utilizing a uniform analytic method in fresh samples, and normalizing against multiple sequences of genomic DNA would be most likely to generate conclusive evidence. As new methodologies for evaluation of mitochondrial function are validated, they should also be explored to see if these methods deliver a more consistent avenue of measuring metabolic activity in developing embryos. The concept of mitochondrial function as an adjunct parameter to help select embryos for successful implantation remains an exciting possibility and continues to generate further investigation.

\section{Declaration of interest}

E $S$ is a consultant for and receives research funding from Foundation for Embryonic Competence. The other author has nothing to disclose.

\section{Funding}

This research did not receive any specific grant from any funding agency in the public, commercial or not-for-profit sector.

\section{Acknowledgement}

Authors would like to thank Man Zhang, PhD for her effort in preparing the figure.

\section{References}

Aitken RJ 2017 Reactive oxygen species as mediators of sperm capacitation and pathological damage. Molecular Reproduction and Development 84 1039-1052. (https://doi.org/10.1002/mrd.22871)

Aitken RJ, Gordon E, Harkiss D, Twigg JP, Milne P, Jennings Z \& Irvine DS 1998 Relative impact of oxidative stress on the functional competence and genomic integrity of human spermatozoa. Biology of Reproduction 59 1037-1046.

Anderson S, Bankier AT, Barrell BG, de Bruijn MH, Coulson AR, Drouin J, Eperon IC, Nierlich DP, Roe BA, Sanger F et al. 1981 Sequence and organization of the human mitochondrial genome. Nature 290 457-465. (https://doi.org/10.1038/290457a0)

Babayev E, Wang T, Szigeti-Buck K, Lowther K, Taylor HS, Horvath T \& Seli E 2016 Aging is associated with changes in mitochondrial dynamics, function and mtDNA quantity. Maturitas 93 121-130. (https://doi. org/10.1016/j.maturitas.2016.06.015)

Birky CW 1995 Uniparental inheritance of mitochondrial and chloroplast genes: mechanisms and evolution. PNAS 92 11331-11338. (https://doi. org/10.1073/pnas.92.25.11331)

Cortopassi GA \& Arnheim N 1990 Detection of a specific mitochondrial DNA deletion in tissues of older humans. Nucleic Acids Research $\mathbf{1 8}$ 6927-6933. (https://doi.org/10.1093/nar/18.23.6927)

Cotterill M, Harris SE, Collado Fernandez E, Lu J, Huntriss JD, Campbell BK \& Picton HM 2013 The activity and copy number of mitochondrial DNA in ovine oocytes throughout oogenesis in vivo and during oocyte maturation in vitro. Molecular Human Reproduction 19 444-450. (https://doi.org/10.1093/molehr/gat013)

Cummins JM 2000 Fertilization and elimination of the paternal mitochondrial genome. Human Reproduction 15 (Supplement 2) 92-101. (https://doi.org/10.1093/humrep/15.suppl_2.92)

Day FR, Ruth KS, Thompson DJ, Lunetta KL, Pervjakova N, Chasman DI, Stolk L, Finucane HK, Sulem P, Bulik-Sullivan B et al. 2015 Large-scale genomic analyses link reproductive aging to hypothalamic signaling, breast cancer susceptibility and BRCA1-mediated DNA repair. Nature Genetics 47 1294-1303. (https://doi.org/10.1038/ng.3412)

de Boer KA, Jansen RRS, Leigh DA \& Mortimer D 1999 Quantification of mtDNA copy number in the human secondary oocyte. Human Reproduction 14 91-92. (https://doi.org/10.1093/humrep/14. Suppl_3.91-a)

Desquiret-Dumas V, Clement A, Seegers V, Boucret L, Ferre-L'Hotellier V, Bouet PE, Descamps P, Procaccio V, Reynier P \& May-Panloup P 2017 The mitochondrial DNA content of cumulus granulosa cells is linked to embryo quality. Human Reproduction 32 607-614. (https://doi. org/10.1093/humrep/dew341)

Diez-Juan A, Rubio C, Marin C, Martinez S, Al-Asmar N, Riboldi M, Diaz-Gimeno P, Valbuena D \& Simon C 2015 Mitochondrial DNA content as a viability score in human euploid embryos: less is better. Fertility and Sterility 104 534.e1-541.e1. (https://doi.org/10.1016/ j.fertnstert.2015.05.022)

Forman EJ, Hong KH, Franasiak JM \& Scott RT 2014 Obstetrical and neonatal outcomes from the BEST Trial: single embryo transfer with 
euploidy screening improves outcomes after in vitro fertilization without compromising delivery rates. American Journal of Obstetrics and Gynecology 210 157.e1-157.e6. (https://doi.org/10.1016/j. ajog.2013.10.016)

Fragouli E, Spath K, Alfarawati S, Kaper F, Craig A, Michel CE, Kokocinski F, Cohen J, Munne S \& Wells D 2015 Altered levels of mitochondrial DNA are associated with female age, aneuploidy, and provide an independent measure of embryonic implantation potential. PLoS Genetics 11 e1005241. (https://doi.org/10.1371/journal.pgen.1005241)

Fragouli E, McCaffrey C, Ravichandran K, Spath K, Grifo JA, Munne S \& Wells D 2017 Clinical implications of mitochondrial DNA quantification on pregnancy outcomes: a blinded prospective non-selection study. Human Reproduction 32 2340-2347. (https://doi.org/10.1093/humrep/ dex292)

Gardner DK \& Schoolcraft WB 1999 In vitro culture of human blastocysts. In Towards Reprodcutive Certainty: Fertility and Genetics Beyond, pp 378-388. Ed R Jansen. Carnforth: Parthenon publishing.

Hamatani T, Falco G, Carter MG, Akutsu H, Stagg CA, Sharov AA, Dudekula DB, VanBuren V \& Ko MS 2004 Age-associated alteration of gene expression patterns in mouse oocytes. Human Molecular Genetics 13 2263-2278. (https://doi.org/10.1093/hmg/ddh241)

Hammond ER, McGillivray BC, Wicker SM, Peek JC, Shelling AN, Stone P, Chamley LW \& Cree LM 2017 Characterizing nuclear and mitochondria DNA in spent embryo culture media: genetic contamination identified. Fertility and Sterility 107 220.e5-228.e5. (https://doi.org/10.1016/j. fertnstert.2016.10.015)

Harton GL, Munne S, Surrey M, Grifo J, Kaplan B, McCulloh DH, Griffin DK, Wells D \& PGD Practitioners Group 2013 Diminished effect of maternal age on implantation after preimplantation genetic diagnosis with array comparative genomic hybridization. Fertility and Sterility $\mathbf{1 0 0}$ 1695-1703. (https://doi.org/10.1016/j.fertnstert.2013.07.2002)

iGenomix Mitoscore 2018 (available at: https://www.igenomix.com/tests/ mitoscore). Accessed on 6 January 2019

Klimczak AM, Pacheco LE, Lewis KE, Massahi N, Richards JP, Kearns WG, Saad AF \& Crochet JR 2018 Embryonal mitochondrial DNA: relationship to embryo quality and transfer outcomes. Journal of Assisted Reproduction and Genetics 35 871-877. (https://doi. org/10.1007/s10815-018-1147-z)

Kort JD, Lathi RB, Brookfield K, Baker VL, Zhao Q \& Behr BR 2015 Aneuploidy rates and blastocyst formation after biopsy of morulae and early blastocysts on day 5. Journal of Assisted Reproduction and Genetics 32 925-930. (https://doi.org/10.1007/s10815-015-0475-5)

Kroener L, Ambartsumyan G, Briton-Jones C, Dumesic D, Surrey M, Munne S \& Hill D 2012 The effect of timing of embryonic progression on chromosomal abnormality. Fertility and Sterility 98 876-880. (https:// doi.org/10.1016/j.fertnstert.2012.06.014)

Lamas-Toranzo I, Pericuesta E \& Bermejo-Alvarez P 2018 Mitochondrial and metabolic adjustments during the final phase of follicular development prior to IVM of bovine oocytes. Theriogenology 119 156-162. (https://doi.org/10.1016/j.theriogenology.2018.07.007)

Leese HJ 2002 Quiet please, do not disturb: a hypothesis of embryo metabolism and viability. BioEssays 24 845-849. (https://doi. org/10.1002/bies.10137)

Luo S, Valencia CA, Zhang J, Lee NC, Slone J, Gui B, Wang X, Li Z, Dell S, Brown J et al. 2018 Biparental inheritance of mitochondrial DNA in humans. PNAS 115 13039-13044. (https://doi.org/10.1073/ pnas.1810946115)

Luoma P, Melberg A, Rinne JO, Kaukonen JA, Nupponen NN, Chalmers RM, Oldfors A, Rautakorpi I, Peltonen L, Majamaa K et al. 2004 Parkinsonism, premature menopause, and mitochondrial DNA polymerase gamma mutations: clinical and molecular genetic study. Lancet 364 875-882. (https://doi.org/10.1016/S01406736(04)16983-3)

Lynch M, Koskella B \& Schaack S 2006 Mutation pressure and the evolution of organelle genomic architecture. Science 311 1727-1730. (https://doi. org/10.1126/science.1118884)

May-Panloup P, Chretien MF, Jacques C, Vasseur C, Malthiery Y \& Reynier P 2005 Low oocyte mitochondrial DNA content in ovarian insufficiency. Human Reproduction 20 593-597. (https://doi.org/10.1093/humrep/ deh667)

Menken J, Trussel J \& Larsen U 1986 Age and infertility. Science 233 1389-1394. (https://doi.org/10.1126/science.3755843)
Monnot S, Samuels DC, Hesters L, Frydman N, Gigarel N, Burlet P, Kebrat V, Lamazou F, Frydman R, Benachi A et al. 2013 Mutation dependence of the mitochondrial DNA copy number in the first stages of human embryogenesis. Human Molecular Genetics 22 1867-1872. (https://doi.org/10.1093/hmg/ddt040)

Muller-Hocker J, Schafer S, Weis S, Munscher C \& Strowitzki T 1996 Morphological-cytochemical and molecular genetic analyses of mitochondria in isolated human oocytes in the reproductive age. Molecular Human Reproduction 2 951-958. (https://doi.org/10.1093/ molehr/2.12.951)

Pasquariello R, Ermisch AF, Silva E, McCormick S, Logsdon D, Barfield JP, Schoolcraft WB \& Krishner RL 2018 Alterations in oocyte mitochondrial number and function are related to spindle defects and occur with maternal aging in mice and humans. Biology of Reproduction Epub. (https://doi.org/10.1093/biolre/ioy248)

Piyamongkol W, Bermudez MG, Harper JC \& Wells D 2003 Detailed investigation of factors influencing amplification efficiency and allele drop-out in single cell PCR: implications for preimplantation genetic diagnosis. Molecular Human Reproduction 9 411-420. (https://doi. org/10.1093/molehr/gag051)

Ramalho-Santos J, Varum S, Amaral S, Mota PC, Sousa AP \& Amaral A 2009 Mitochondrial functionality in reproduction: from gonads and gametes to embryos and embryonic stem cells. Human Reproduction Update 15 553-572. (https://doi.org/10.1093/humupd/dmp016)

Ravichandran K, McCaffrey C, Grifo J, Morales A, Perloe M, Munne S, Wells D \& Fragouli E 2017 Mitochondrial DNA quantification as a tool for embryo viability assessment: retrospective analysis of data from single euploid blastocyst transfers. Human Reproduction 32 1282-1292. (https://doi.org/10.1093/humrep/dex070)

Reynier P, May-Panloup P, Chretien MF, Morgan CJ, Jean M, Savagner F, Barriere P \& Malthiery Y 2001 Mitochondrial DNA content affects the fertilizability of human oocytes. Molecular Human Reproduction 7 425-429. (https://doi.org/10.1093/molehr/7.5.425)

Sanchez T, Wang T, Pedro MV, Zhang M, Esencan E, Sakkas D, Needleman D \& Seli E 2018 Metabolic imaging using Flurescence Lifetime Imaging Microscopy (FLIM) accurately detects mitochondrial dysfunction in mouse oocytes. Fertility and Sterility 110 1387-1397. (https://doi.org/10.1016/j.fertnstert.2018.07.022)

Seli E 2016 Mitochondrial DNA as a biomarker for in-vitro fertilization outcome. Current Opinion in Obstetrics and Gynecology 28 158-163. (https://doi.org/10.1097/GCO.0000000000000274)

Society for Assisted Reproductive Technology. National Summary Report 2016. (available at: https://www.sartcorsonline.com/rptCSR_ PublicMultYear.aspx? reportingYear=2016). Accessed on 6 January 2019.

Stigliani S, Anserini P, Venturini PL \& Scaruffi P 2013 Mitochondrial DNA content in embryo culture medium is significantly associated with human embryo fragmentation. Human Reproduction 28 2652-2660. (https://doi.org/10.1093/humrep/det314)

Stigliani S, Persico L, Lagazio C, Anserini P, Venturini PL \& Scaruffi P 2014 Mitochondrial DNA in day 3 embryo culture medium is a novel, non-invasive biomarker of blastocyst potential and implantation outcome. Molecular Human Reproduction 20 1238-1246. (https://doi. org/10.1093/molehr/gau086)

Sutovsky P, Navara CS \& Schatten G 1996 Fate of the sperm mitochondria and the incorporation, conversion and disassembly of the sperm tail structures during bovine fertilization. Biology of Reproduction $\mathbf{5 5}$ 1195-1205. (https://doi.org/10.1095/biolreprod55.6.1195)

Taanman JW 1999 The mitochondrial genome: structure, transcription, translation and replication. Biochimica et Biophysica Acta $\mathbf{1 4 1 0}$ 103-123. (https://doi.org/10.1016/S0005-2728(98)00161-3)

Toner JP 2002 Progress we can be proud of: U.S. trends in assisted reproduction over the first 20 years. Fertility and Sterility 78 943-950. (https://doi.org/10.1016/S0015-0282(02)04197-3)

Treff NR, Zhan Y, Tao X, Olcha M, Han M, Rajchel J, Morrison L, Morin S] \& Scott RT 2017 Levels of trophectoderm mitochondrial DNA do not predict the reproductive potential of sibling embryos. Human Reproduction 32 954-962. (https://doi.org/10.1093/humrep/dex034)

Trifunovic A, Wredenberg A, Falkenberg M, Spelbrink JN, Rovio AT, Bruder CE, Bohlooly M, Gidlof S, Oldfors A, Wibom R et al. 2004 Premature ageing in mice expressing defective mitochondrial DNA polymerase. Nature 429 417-423. (https://doi.org/10.1038/ nature02517) 
Victor AR, Brake AJ, Tyndall JC, Griffin DK, Zouves CG, Barnes FL \& Viotti M 2017 Accurate quantitation of mitochondrial DNA reveals uniform levels in human blastocysts irrespective of ploidy, age, or implantation potential. Fertility and Sterility 107 34.e3-42.e3. (https:// doi.org/10.1016/j.fertnstert.2016.09.028)

Wang T, Babayev E, Jiang Z, Li G, Zhang M, Esencan E, Horvath T \& Seli E 2018 Mitochonrdial unfolded protein response gene Clpp is required to maintain ovarian follicular reserve during aging, for oocyte competence, and development of pre-implantation embryos. Aging Cell 30 e12784. (https://doi.org/10.1111/acel.12784)

Wells D 2017 Mitochondrial DNA quantity as a biomarker for blastocyst implantation potential. Fertility and Sterility 108 742-747. (https://doi. org/10.1016/j.fertnstert.2017.10.007)
Wilding M, Dale B, Marino M, di Matteo L, Alviggi C, Pisaturo ML, Lombardi L \& De Placido G 2001 Mitochondrial aggregation patterns and activity in human oocytes and preimplantation embryos. Human Reproduction 16 909-917. (https://doi.org/10.1093/humrep/16.5.909)

Received 9 November 2018

First decision 17 December 2018

Revised manuscript received 26 February 2019

Accepted 7 March 2019 